\title{
Hybrid Model Predictive Control for Optimal Energy Management of a Smart House
}

\author{
Albina Khakimova, \\ Akmaral Shamshimova, \\ Dana Sharipova, \\ Aliya Kusatayeva, \\ Viktor Ten \\ Nazarbayev University Research \\ and Innovation System (NURIS) \\ Astana, Kazakhstan \\ Email: \{akhakimova,akmaral.shamshimova, \\ dana.sharipova,vten\}@nu.edu.kz

\begin{abstract}
This paper describes the modeling and control of heat and electricity flows in a smart house equipped with a solar heating system, PV panels, and lead-acid batteries for energy storage. The goal is to minimize electricity costs, making best use of renewable sources of heat and electricity. The system model is obtained via system identification from experimental data as a discrete-time hybrid system to capture the main thermal and electrical dynamics, the on-off activation of pumps, heating coil, the connection to the grid, and various operating constraints, including logic constraints and limits on system variables. Based on the obtained model, we derive a hybrid model predictive control (MPC) strategy. The controller is able to track the desired temperature and minimize costs for consuming electricity from the grid, while respecting all the prescribed constraints. Simulation results testify the effectiveness and feasibility of the approach.
\end{abstract}

\author{
Alberto Bemporad \\ IMT Institute for Advanced Studies \\ Lucca, Italy \\ Email: alberto.bemporad@imtlucca.it
}

Dept. of

\section{INTRODUCTION}

The aim of this paper is to design an intelligent energy management system for a prototype "smart house" (Fig. 1). The electrical power to operate the appliances can be drawn from the grid, or from a battery pack, charged through PV panels. On the other hand, the house can be heated by solar collectors and by an electrical heating coil. The management system must be able to satisfy the demand of electrical power from the electrical loads while maintaining the inside temperature in a desired interval, and minimizing the overall consumption of energy sourced from the grid, also taking its price into account. A time-varying energy price is usually imposed in order to push the consumers to use energy mainly when there is availability on the global scale. Therefore, a smart house that draws less power from the grid when the price is high can help in reducing the overall energy production from centralized power plants, and leads to a better exploitation of local renewable sources. The presence of binary control variables (electrical heater on/off, pumps on/off, electrical load powered from the grid or from the battery pack) makes the process a hybrid dynamical system [1], [2], containing both physical components (the evolution of which is described by difference equations) and logical components.

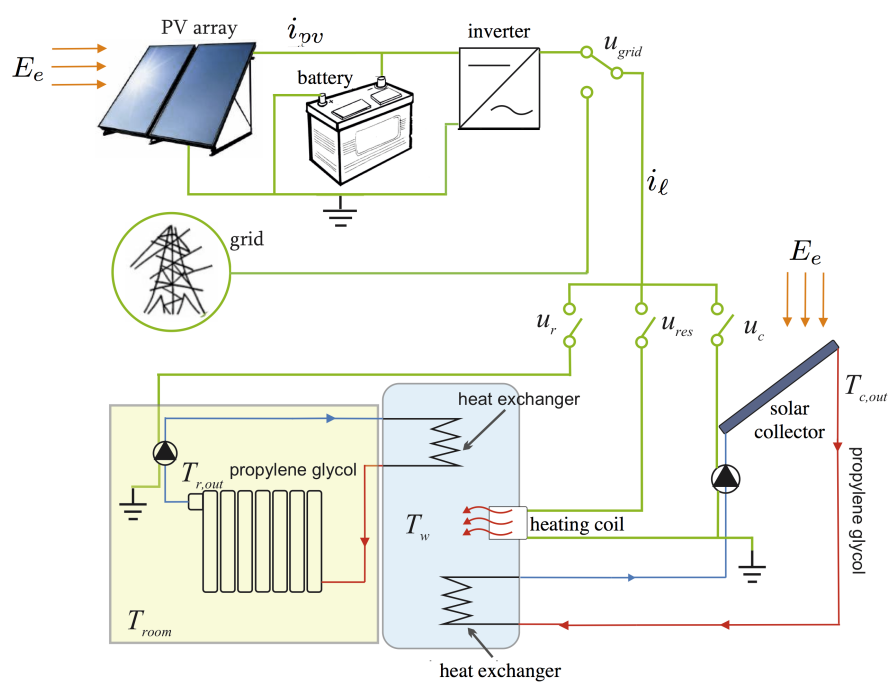

Fig. 1. Electrical and thermal subsystems of the smart house

The energy management approach presented in this paper needs first to obtain a control-oriented state-space dynamical model of the system, based on real data from the experimental site. The electrical part mainly describes the evolution of the battery state of charge as a function of different current terms, under given assumptions. The thermal model, describing the coupled evolutions of four different temperatures in the smart house, depending on manipulated and disturbance inputs (such as ambient temperature and solar radiation) is obtained via grey-box identification. When dealing with control algorithms that make use of thermal models aimed at predictions, it is of essential importance to obtain models that are simple enough to be employed for real-time computations, at the same time describing the thermal dynamics of the building with sufficient accuracy. In many recent works, the focus has been on modeling large buildings, where many sources of heating/cooling are taken into account (see, among others, [3][5]), while our focus will be on a small-scale system. 
The presence of conflicting goals, constraints, and the possibility to exploit model-based predictions of the system dynamics naturally leads to consider optimal control strategies, and, more specifically, model predictive control (MPC), which is one of the most successful advanced control techniques (see [6] for an overview). MPC is a class of advanced control methodologies, first introduced in chemical plants, which are able to cope with multivariable plants with coupled dynamics, and constraints on input and state variables. Determining the control actions at each sampling instant requires solving online a constrained optimization problem. Since such a problem can be computationally expensive, MPC has been traditionally applied to systems with relatively large sampling intervals (e.g., minutes) [7], [8]. In the last years, thanks to improved numerical algorithms and better computational capabilities, the use of MPC has also been extended to systems with fast dynamics [9]-[11]. In this work, due to the hybrid nature of the considered system, the MPC control law requires the online solution of a mixed-integer optimization problem (see, among others, [12]) at each sampling instant. Many different approaches have been proposed for MPC of hybrid systems, but the main ideas can be found in the seminal paper [13].

The application of MPC for building automation, as opposed to rule-based systems, is becoming an extremely popular research topic. To the best of our knowledge, all the proposed approaches take into account the modeling and control of large-scale buildings, where many sources of heating/cooling are considered [14]-[20]. As a result, a performance improvement has been observed with respect to traditional rule-based control systems. On the other hand, the control of the electrical energy flow is usually considered for very large-scale and distributed systems (smart grids), see, e.g., [21]. Different types of algorithms are required for the combined decision on how to actuate the heating system and manage the electrical energy flow from renewables. Such a challenge is addressed in this paper for an experimental small-scale building.

The paper is structured as follows: Section II describes the model of the electrical and the thermal subsystems that compose the smart building. The procedure for the synthesis of the MPC controller is introduced and discussed in Section III, while the related simulation results are shown in Section IV. Conclusions are drawn in Section V.

\section{SYSTEM MODELING AND IDENTIFICATION}

\section{A. Electrical subsystem}

The electrical subsystem of the smart house (Fig. 2) consists of PV panels, a battery pack, an inverter, and a transfer switch. At full illumination, the PV array (14 solar modules Alfasolar Pyramid 60P/250) is capable of supplying power to the smart house and charging the attached lead-acid battery pack. The battery pack consists of eight lead-acid batteries Effekta BTL 12-200 [22], with a nominal voltage of $12 \mathrm{~V}$, and a nominal capacity of $200 \mathrm{Ah}$. The connections are such that the battery pack has a total capacity $Q=800 \mathrm{Ah}$ and a nominal voltage $V_{b}=24 \mathrm{~V}$ (see Fig. 2), and the common PV-Battery DC-bus is connected to an inverter. The load can be powered directly from a utility terminal (grid) or from the inverter through a transfer switch. The latter can only assume two configurations, represented by the input variable $u_{\text {grid }} \in\{0,1\}$ : connecting the load to the grid $\left(u_{\text {grid }}=1\right)$ or to the inverter $\left(u_{\text {grid }}=0\right)$. The present setting does not allow the use of the inverter as a battery charger when solar power is not available, since it is impossible to connect the inverter to the utility terminal by the transfer switch.

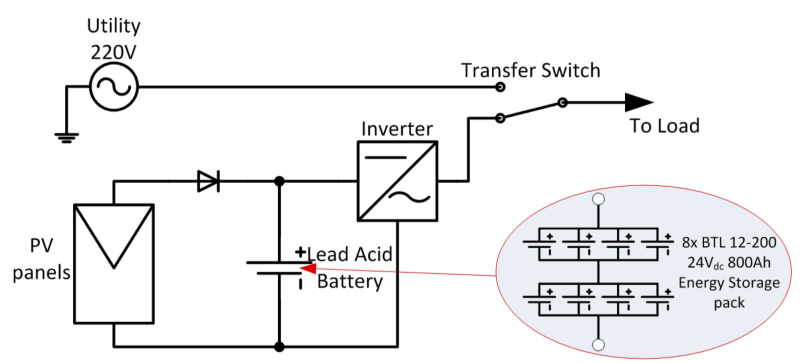

Fig. 2. Schematic diagram of the smart house electrical subsystem

The design and implementation of the proposed hybrid MPC control law for the smart house requires a model, in which the different components have linear or on/off behavior. However, most of real components such as PV panels, batteries, and power converters are nonlinear in nature. Nonetheless, for a given operating mode and under certain assumptions, a linear model can represent a sufficient approximation of the real behavior, at least for control design purposes. The following assumptions are made: the discharge power capability of the battery pack is much higher than the maximum output power of the PV array, and the useful energy of the battery pack (product of total energy by depth of discharge) can be extracted in a range of state of charge in which the terminal voltage of the battery pack is approximately constant. The first assumption allows one to claim that the voltage of the DC bus is imposed by the state of charge of the battery pack. The second assumption is based on the manufacture's recommendations to keep the depth of discharge as low as possible to increase battery life.

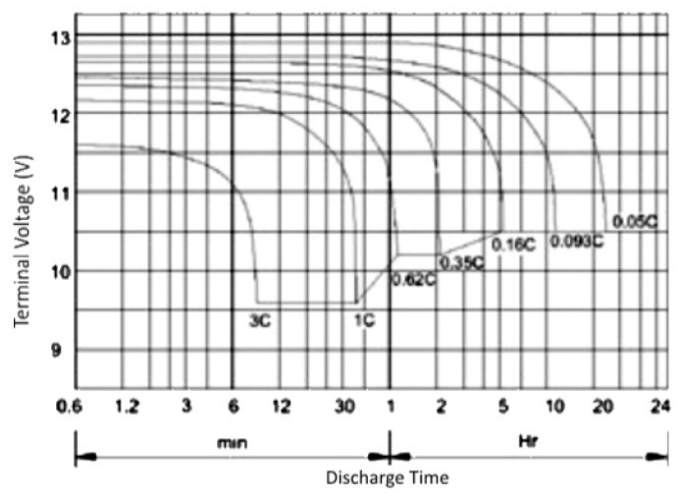

Fig. 3. Lead-acid battery terminal voltage as a function of C-rate and discharge time (equivalent of state of charge), from the product data sheet ${ }^{2}$.

As a result, as can be seen in Fig. 3, for any rate of discharge (C-rate), the battery voltage is almost constant if the discharge ends before the state of charge becomes excessively low. If these two assumptions are reasonable, the output current 
of the PV array, as can be easily observed in the V-I curves in the product data sheet [23], is linearly proportional to the solar irradiance $E_{e}$ for a constant voltage at the PV terminals. In order to find the coefficient relating the two variables, the value of $i_{\mathrm{pv}}$ generated by the PV array has been measured, together with the solar irradiance $E_{e}$ during normal operation of the system. As a result, by linear regression we obtained

$$
i_{\mathrm{pv}} \simeq 0.1218 \cdot E_{e}
$$

Under the given assumptions, it is possible to describe the time evolution of the state of charge (referred to as $S$ in the following) for the whole battery pack, by using the following difference equations:

$$
S(k+1)= \begin{cases}S(k)+\frac{T_{s}}{Q} i_{\mathrm{pv}}(k) & \text { if } u_{\text {grid }}=1 \\ S(k)+\frac{T_{s}}{Q}\left(i_{\mathrm{pv}}(k)-i_{\ell}(k)\right) & \text { if } u_{\text {grid }}=0\end{cases}
$$

in which the index $k$ represents the discrete time instant, with a sampling time $T_{s}=10$ minutes. Also, $i_{\mathrm{pv}}$ and $i_{\ell}$ are meant to represent the average value of the current generated by the PV array and the current absorbed by the load in the considered sampling interval. The appliances in the house consist of an electrical heating coil (with power consumption equal to $P_{r e s}=2 \mathrm{~kW}$ ), two pumps (collector and radiator pumps, with power consumption equal to $P_{c}=P_{r}=0.3 \mathrm{~kW}$, respectively), and additional utilities, such as computers (with power consumption assumed constant and equal to $P_{u}=0.3$ $\mathrm{kW})$. The purpose of the two pumps will be explained in the next subsection. While the additional utilities are considered always on, the electrical heating coil and the pumps can be switched on/off by the control system. For this reason, we define 3 additional binary variables, analogous to $u_{\text {grid }}$, as follows:

$$
\begin{aligned}
u_{\mathrm{res}} & = \begin{cases}1 & \text { if electrical heating coil on } \\
0 & \text { if electrical heating coil off, }\end{cases} \\
u_{c} & = \begin{cases}1 & \text { if collector pump on } \\
0 & \text { if collector pump off, }\end{cases} \\
u_{r} & = \begin{cases}1 & \text { if radiator pump on } \\
0 & \text { if radiator pump off. }\end{cases}
\end{aligned}
$$

As a result, the value of $i_{\ell}$ at a given time instant $k$ can be expressed as

$$
i_{\ell}(k)=\frac{1}{V_{b}}\left(P_{r e s} u_{\mathrm{res}}(k)+P_{c} u_{c}(k)+P_{r} u_{r}(k)+P_{u}\right) .
$$

Also, we express the overall power consumption as $P_{\ell}=$ $P_{\text {res }}+P_{c}+P_{r}+P_{u}$. In addition to $E_{\mathrm{e}}$ and $i_{\mathrm{pv}}$, a third external signal has to be considered, that is the time-varying price of electricity $q_{\mathrm{e}}[\mathrm{c} € / \mathrm{kWh}$. This value is of fundamental importance for the control strategy, that aims at minimizing the overall expense, while enforcing the system variables to remain in given intervals. We consider a realistic scenario taking into account the two-rate tariff

$$
q_{\mathrm{e}}= \begin{cases}q_{e, d} & \text { between } 7 \text { a.m. and } 11 \text { p.m. } \\ q_{e, n} & \text { between } 11 \text { p.m. and } 7 \text { a.m. }\end{cases}
$$

where $q_{e, d}=10.5 \mathrm{c} € / \mathrm{kWh}$, while $q_{e, n}=5.6 \mathrm{c} € / \mathrm{kWh}$. Given a sequence of $\bar{N}$ electricity price values $q_{\mathrm{e}}(k)$, sampled with time interval $T_{s}=10$ minutes, and average power consumption $P_{\ell}(k)$ (expressed in $\mathrm{W}$ ) for all samples, we obtain the overall expense in $c €$

$$
\sum_{k=0}^{\bar{N}-1} \alpha P_{\ell}(k) q_{\mathrm{e}}(k) u_{\text {grid }}(k), \quad \alpha=10^{-3} \cdot \frac{T_{s}}{60} .
$$

Notice that (5) accounts for the fact that, during the time intervals when we are drawing power from the battery (i.e., when $u_{\text {grid }}=0$ ), the expense is equal to zero. The use of a specific tariff does not limit the validity of our approach, which can be applied to any time-varying electricity price that is known a priori. In fact, $q_{e}$ will be considered by the MPC controller as a measured external input, with known future evolution.

\section{B. Thermal subsystem}

The solar heating system consists of evacuated tubed solar collectors, thermal tank, radiators, circulating pumps, electrical heating coil, and temperature sensors mounted at different points. The system is sketched in Fig. 1. Two types of sensors (PT1000 RTD and NTC10K) have been placed to measure the state variables (temperatures in ${ }^{\circ} C$ ) of the thermal subsystem: the outlet temperature from the solar collector $T_{\mathrm{c} \text {,out }}$, the outlet temperature from the radiator $T_{\mathrm{r} \text {,out }}$, the average temperature in the water tank $T_{\mathrm{w}}$, and the average room temperature $T_{\text {room }}$.

The solar collector consists of 20 vacuum heating tubes with propylene glycole liquid inside. For modeling purposes, the temperature of the evacuated tubes is considered as an average of $T_{\mathrm{c} \text {,out }}$ and $T_{\mathrm{c}, \text { in }}$, this latter being the inlet temperature of the solar collector. Also, the heat capacity of the heating elements inside the thermal tank has been observed to be much smaller than that of the fluid flowing inside them: as a consequence, all power supplied to the heating elements of the heat exchangers goes directly into the fluid in the tank with no delay. Also, the temperature of the fluid inside the tank is approximated as uniform. The temperature of the fluid inside the radiator is considered as an average of $T_{\mathrm{r} \text {,out }}$ and $T_{\mathrm{r}, \text { in }}$, this latter being the inlet temperature of the radiator, and we assume that no heat losses are present in the pipes connecting the tank with the radiator.

For the thermal subsystem, let the vector of state variables $x_{t h}=\left[\begin{array}{llll}T_{\mathrm{c}, \text { out }} & T_{\mathrm{w}} & T_{\mathrm{r}, \text { out }} & T_{\text {room }}\end{array}\right]^{\prime}$, and the disturbance input vector $d_{t h}=\left[\begin{array}{ll}T_{\mathrm{amb}} & E_{\mathrm{e}}\end{array}\right]^{\prime}$, in which $E_{\mathrm{e}}\left[\mathrm{W} / \mathrm{m}^{2}\right]$ is the solar irradiance (already mentioned for the electrical subsystem) and $T_{a m b}\left[{ }^{\circ} \mathrm{C}\right]$ is the ambient (external) temperature. Considering that the manipulated input variables of the thermal subsystem are $u_{r}, u_{c}$, and $u_{\text {res }}$, we obtain the following discrete-time model structure from physical considerations, again with sampling time $T_{s}=10$ minutes:

$x_{t h}(k+1)=A\left(u_{r}(k), u_{c}(k)\right) x_{t h}(k)+B u_{\mathrm{res}}(k)+E d_{t h}(k)$

where

$$
\begin{aligned}
A\left(u_{r}, u_{c}\right) & =\left[\begin{array}{cccc}
a_{11}\left(u_{c}\right) & a_{12}\left(u_{c}\right) & 0 & 0 \\
a_{21} & a_{22} & a_{23} & a_{24} \\
0 & a_{32}\left(u_{r}\right) & a_{33}\left(u_{r}\right) & a_{34} \\
0 & a_{42} & a_{43} & a_{44}
\end{array}\right] \\
B & =\left[\begin{array}{c}
0 \\
b_{2} \\
0 \\
0
\end{array}\right], \quad D=\left[\begin{array}{cc}
e_{11} & e_{12} \\
0 & 0 \\
0 & 0 \\
e_{41} & e_{42}
\end{array}\right]
\end{aligned}
$$


Notice that, since some elements of $A\left(u_{r}, u_{c}\right)$ depend either on $u_{c}$ or on $u_{r},(6)$ is not a linear model, because of the change in the heat transmission coefficients due to fluid circulation. However, given each of the four possible combinations of $\left(u_{c}, u_{r}\right) \in\{0,1\} \times\{0,1\}$, it is possible to obtain one of the corresponding constant matrices $A(0,0), A(0,1), A(1,0)$, $A(1,1)$. Systems of this kind are called switched linear systems [24], and represent a particular form of hybrid dynamics. Determining the coefficients of all realizations of $A\left(u_{r}, u_{c}\right)$, and of $B$ and $E$ from physical principles would not account for the parameter mismatches due to the wear of the components, or to unmodeled effects. The matrix structure (i.e., zero or non-zero elements) for all configurations is used to obtain a discrete-time model from experimental data. Advanced statespace identification methods such as subspace identification methods are not needed in this case, since all states and inputs are measured in the experimental facility. As a consequence, a simple linear regression is sufficient to obtain all the needed parameters. As for the experiment design, the control actions have been defined so as to make the four temperatures oscillate in all the range of frequencies in which the system can be excited, by acting on $u_{\text {res }}$, while the pumps are turned on and off at regular time intervals, in order to obtain data for all four realizations of $A\left(u_{r}, u_{c}\right)$. The fact of inferring the matrix structure from physical principles. and obtaining the parameters from experimental data, makes this a grey-box system identification process [25].

\section{Hybrid model of the overall system}

Overall, by collecting equations (2), (3), and (6), we define $x \in \mathbb{R}^{5}$ (state vector), $u \in \mathbb{R}^{4}$ (manipulable input vector) and $d \in \mathbb{R}^{5}$ (uncontrolled input vector) as

$$
x=\left[\begin{array}{c}
T_{\mathrm{c}, \text { out }} \\
T_{\mathrm{w}} \\
T_{\mathrm{r}, \mathrm{out}} \\
T_{\mathrm{room}} \\
S
\end{array}\right], u=\left[\begin{array}{c}
u_{c} \\
u_{r} \\
u_{\mathrm{res}} \\
u_{\text {grid }}
\end{array}\right], d=\left[\begin{array}{c}
T_{\mathrm{amb}} \\
E_{\mathrm{e}} \\
i_{\mathrm{pv}} \\
P_{u} \\
q_{e}
\end{array}\right] .
$$

All the previously-considered equations of the system dynamics can be written in the concise form

$$
x(k+1)=f(x(k), u(k), d(k))
$$

which includes linear and switched-linear dynamics, in which the manipulated inputs are binary variables. Models of this kind can be formally expressed as discrete hybrid automata (DHA) [26], which constitute a very versatile modeling framework for linear hybrid systems. In order to implement the MPC control code, the DHA model is translated into an equivalent hybrid model described by linear mixed-integer equalities and inequalities, called mixed logical dynamical (MLD) systems, introduced in [13]. In this paper, the formal description of how to obtain the equivalent MLD description of (8) will be omitted due to space limitation. The hybrid systems modeling language HYSDEL introduced in [26] is used to describe model (8) as a DHA and to automatically transform it in MLD form. The reader is referred to [13], [26] for the detailed procedure in the general framework.

\section{HYBRID MODEL PREDICTIVE CONTROLLER}

The idea behind MPC is to start with a model of the openloop process that explains the dynamical relations among the variables of the system (manipulated and uncontrolled inputs, and state variables). Then, constraint specifications on system variables are added, such as input limitations (typically due to actuator saturation) and desired bounds that the state variables should not exceed. Desired performance specifications complete the control problem setup and are expressed through different weights on tracking errors and actuator efforts (as in classical linear quadratic regulation). At each sampling time, an open-loop optimal control problem based on the given model, constraints, weights, and with initial condition set at the current (measured or estimated) value of the state, is repeatedly solved through numerical optimization. The result of the optimization is an optimal sequence of future control moves. Only the first sample of such a sequence is actually applied to the process; the remaining moves are discarded. At the next time step, a new optimal control problem based on new measurements is solved over a shifted prediction horizon.

MPC based on hybrid dynamical models has emerged as a very promising approach to handle switching linear dynamics, on/off inputs, logic states, as well as logic constraints on input and state variables [13], [27], [28]. Hybrid MPC design is a systematic approach to meet performance and constraint specifications in spite of the complexity due to the interaction between continuous and logic dynamics.

At each sampling instant, after reading the measured values of temperatures and battery state of charge, the MPC controller determines the sequence of inputs that leads to the best possible behavior of the system over a prediction horizon of $N$ sampling intervals. A generic planned input sequence is referred to as $\mathbf{u}=\left\{u_{0} u_{1} \ldots u_{N-1}\right\}$, in which every element represent a possible realization of vector $u$ for a specific time instant. The input sequence determining the optimal behavior will be referred to as $\mathbf{u}^{*}=\left\{\begin{array}{llll}u_{0}^{*} & u_{1}^{*} \ldots & u_{N-1}^{*}\end{array}\right\}$. The cost function will be analogous to the overall electricity cost in (5), with the difference that it will refer to a particular prediction, rather than on measured values. From the practical point of view, this means that we want to find the input sequence in a given window in the future that minimizes the expense in that time window, assuming perfect knowledge of the system model and of the uncontrolled input $d$.

Regarding the future values of vector $d$, the time evolution of electricity price $q_{\mathrm{e}}$ and power consumption of additional utilities $P_{u}$ are known in advance, being the first expressed in (5), and the second assumed constant for simplicity. As for $E_{\mathrm{e}}$ and $T_{\mathrm{amb}}$, we need to rely on a weather forecast (cf. [29]). Since the forecast values at a given time instant will be in general different from the actual ones, we refer to the forecast ones as $\tilde{E}_{e}$ and $\tilde{T}_{\mathrm{amb}}$, respectively, always considering that the forecast value is the one available when the control action is computed. Finally, the forecast current $\tilde{i}_{p v}$ will be obtained as a function of the forecast solar irradiance $\tilde{E}_{e}$.

In this way, given the weather forecast data, we can generate a prediction, for every time instant in the prediction horizon, of the forecast vector of uncontrolled inputs $\tilde{d}=\left[\begin{array}{lllll}\tilde{T}_{\mathrm{amb}} & \tilde{E}_{e} & \tilde{i}_{p v} & P_{u} & q_{e}\end{array}\right]^{\prime}$. 
The final element that needs to be taken into account consists of the imposed constraints on state and input variables. As for the state variables, we express the inclusion of a state vector $x$ in the set of feasible state values $\mathcal{X}$ as follows:

$$
x \in \mathcal{X} \Leftrightarrow\left\{\begin{aligned}
T_{\text {room }} & \in\left[20^{\circ} \mathrm{C}, 21^{\circ} \mathrm{C}\right] \\
T_{\mathrm{c}, \text { out }} & \in\left[-5^{\circ} \mathrm{C}, 120^{\circ} \mathrm{C}\right] \\
T_{\mathrm{w}} & \in\left[3^{\circ} \mathrm{C}, 80^{\circ} \mathrm{C}\right] \\
T_{\mathrm{r}, \text { out }} & \in\left[3^{\circ} \mathrm{C}, 80^{\circ} \mathrm{C}\right] \\
S & \in[30 \%, 80 \%]
\end{aligned}\right.
$$

The optimal control problem to be solved at every sampling time can be expressed as

$$
\begin{aligned}
& \min _{\mathbf{u}} \sum_{k=0}^{N-1} \alpha P_{\ell, k} \cdot q_{e, k} \cdot u_{\text {grid }, k} \\
& \text { s.t. }\left\{\begin{aligned}
x_{0} & =x(k) \\
x_{k+1} & =f\left(x_{k}, u_{k}, \tilde{d}_{k}\right), k=0, \ldots, N-1 \\
x & \in \mathcal{X}, k=1, \ldots, N
\end{aligned}\right.
\end{aligned}
$$

According to the receding horizon principle, only the first value of the sequence is applied, i.e., $u(k)=u_{0}^{*}$, and a new optimal sequence is recomputed at the next sampling instant. The state constraints defined by set $\mathcal{X}$ are not enforced as hard constraints, but rather as soft constraints. That is to say, if no feasible solution is found for which all constraints are satisfied, the MPC controller will find the sequence that minimizes the amount of constraint violation, since a penalty term is added in the cost function to penalize constraint violation.

\section{Closed-LOOP SIMULATION RESUlTS}

In order to test the proposed MPC controller, which has been implemented using the Hybrid Toolbox [30], a simulation example over a period of 5 days is presented, and the results are shown in Figs. 4, 5, and 6. The values of $E_{e}$ and $T_{a m b}$ are obtained from measured data during the month of December in the experimental site. For the sake of simplicity, in the presented simulation the forecasted values $\tilde{E}_{e}$ and $\tilde{T}_{a m b}$ coincide with the measured ones. The other external inputs are the constant power consumption of the additional utilities $P_{u}=0.3 \mathrm{~kW}$, the current produced by the PV array, obtained from $E_{e}$ via equation (1), and the electricity price $q_{e}$ in (4). In order to allow the use of a rather long prediction horizon, equal to 8 hours, the MPC controller implements the equivalent version of the described model for prediction (i.e., (10b)) with sampling time $\bar{T}_{s}=30$ minutes (as a consequence, $N=16)$. However, the control action is updated with an interval $T_{s}=10$ minutes, which allows the controller to quickly recover from the effect of unmodeled dynamics or erroneous forecasts of the uncertain terms.

Considering the thermal subsystem, the controller is always taking action to prevent constraints violation, considering the changes in the allowable range with 8 hours notice (Fig. 6). Only relatively small violations of the imposed lower bound on $T_{\text {room }}$ are observed. In particular, notice that in the 5 th day the ambient temperature $T_{a m b}$ drops from around $0^{\circ} \mathrm{C}$ to around $-20^{\circ} \mathrm{C}$, but the room temperature is kept in the desired range, thanks to the predictive action of the controller. Notice that all the other constraints on the temperatures are satisfied.

As for the electrical subsystem, the constraints on the state of charge value are also enforced, which prevents the battery
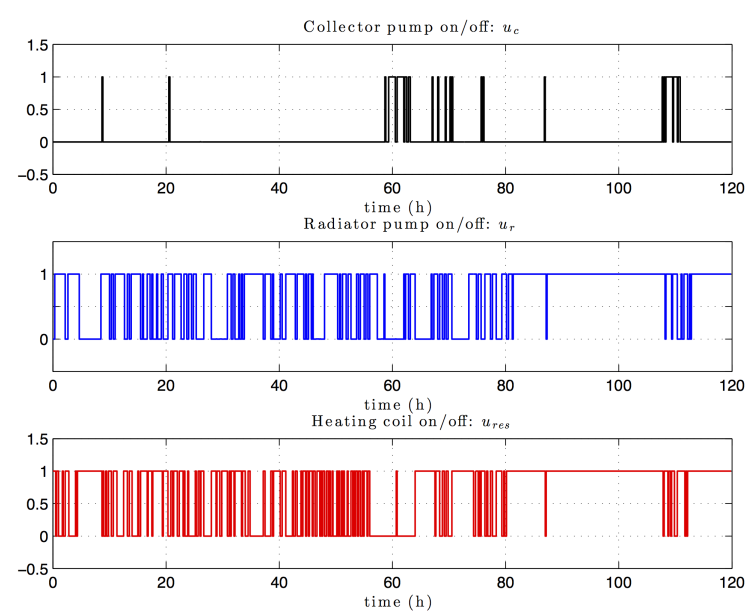

Fig. 4. Time evolution of the binary inputs $u_{c}, u_{r}$, and $u_{\text {res }}$
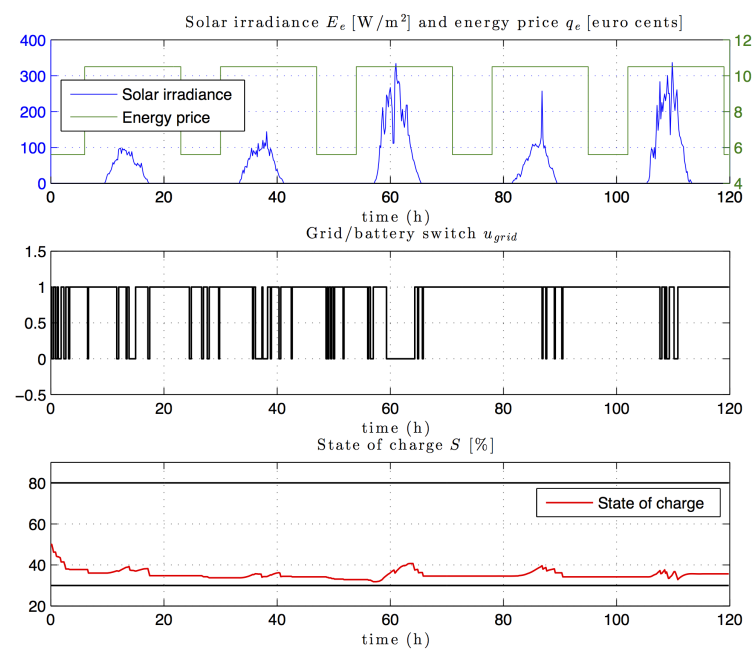

Fig. 5. Time evolution of $E_{e}, q_{e}, u_{\text {grid }}$, and $S$
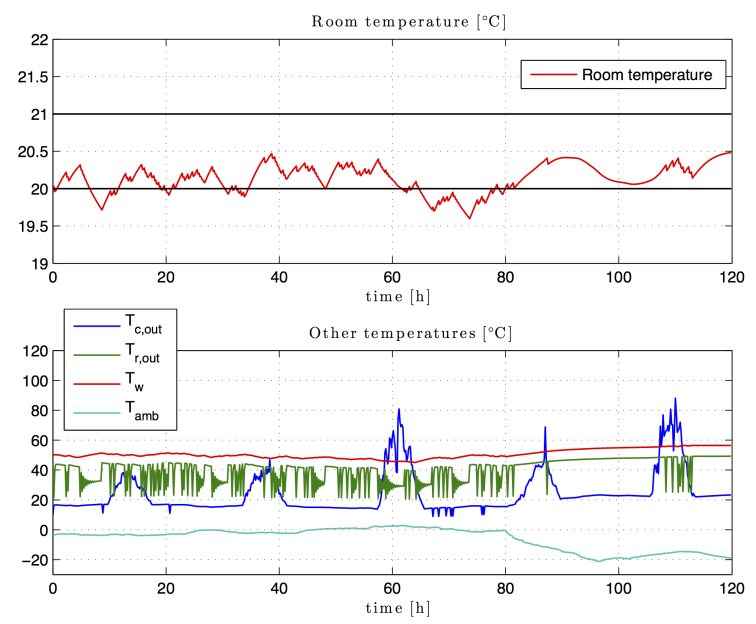

Fig. 6. Upper figure: time evolution of $T_{\text {room. Lower figure: time evolution }}$ of states $T_{c, \text { out }}, T_{r, \text { out }}, T_{w}$, and disturbance input $T_{a m b}$ 
from being damaged. Also, when the night tariff is applied, the energy from the grid is mostly preferred, in order to save the energy stored in the battery for the day, when the price of electricity will be higher. This is automatically obtained if the prediction horizon is long enough to calculate the effect of the day tariff, when the control action is decided during the night.

The simulations were run on a $3.2 \mathrm{GHz}$ Intel Core i3 processor with 4GB RAM, using MATLAB. The MILP to be considered at each sampling instant is solved with GUROBI [12]. The recorded computation time has an average value of $3.78 \mathrm{~s}$, with a worst-case value of $57.23 \mathrm{~s}$. This allows one to neglect the computation time with respect to the sampling interval $T_{s}$, and makes the approach feasible for the foreseen experimental implementation.

\section{CONCLUSIONS AND OUTLOOK}

In this paper, we set up a linear hybrid model of a smart house and a model predictive controller that smoothly coordinates on/off pumps, on/off heating coil activation, on/off switch between grid and battery, and is able to minimize electricity costs, exploiting future information on exogenous inputs. The results, though preliminary, are promising, since they prove the feasibility of the approach and the capabilities of the controller. Future work will include the use of real-time data from weather forecast, the comparison with rule-based systems to assess the actual improvement brought by MPC, and the implementation and testing of the overall control system on our experimental site.

\section{ACKNOWLEDGMENT}

This work has been partially funded by the Ministry of Education and Science of Kazakhstan through the program "Research and development of energy efficiency and energy saving, renewable energy and environmental protection for 2014 - 2016", under project n. 8 ("Integration, Automation and Control of Renewable Power Sources" - "Energy-Efficient Building Management via Model Predictive Control"). The authors also gratefully acknowledge the financial support by BG International Limited.

\section{REFERENCES}

[1] A. J. Van Der Schaft and J. M. Schumacher, An introduction to hybrid dynamical systems. Springer London, 2000.

[2] R. Goebel, R. G. Sanfelice, and A. R. Teel, "Hybrid dynamical systems," IEEE Control Systems Magazine, vol. 29, no. 2, pp. 28-93, 2009.

[3] Y. Ma, F. Borrelli, B. Hencey, B. Coffey, S. Bengea, and P. Haves, "Model predictive control for the operation of building cooling systems," IEEE Transactions on Control Systems Technology, vol. 20, no. 3, pp. 796-803, 2012.

[4] S. Goyal and P. Barooah, "A method for model-reduction of nonlinear thermal dynamics of multi-zone buildings," Energy and Buildings, vol. 47, pp. 332-340, 2012.

[5] S. Goyal, H. A. Ingley, and P. Barooah, "Occupancy-based zone-climate control for energy-efficient buildings: Complexity vs. performance," Applied Energy, vol. 106, pp. 209-221, 2013.

[6] D. Mayne and J. Rawlings, Model Predictive Control: Theory and Design. Madison,WI: Nob Hill Publishing, LCC, 2009.

[7] J. W. Eaton and J. B. Rawlings, "Model-predictive control of chemical processes," Chemical Engineering Science, vol. 47, no. 4, pp. 705-720, 1992.
[8] S. J. Qin and T. A. Badgwell, "A survey of industrial model predictive control technology," Control engineering practice, vol. 11, no. 7, pp. 733-764, 2003.

[9] S. Di Cairano, D. Yanakiev, A. Bemporad, I. V. Kolmanovsky, and D. Hrovat, "Model predictive idle speed control: Design, analysis, and experimental evaluation," IEEE Transactions on Control Systems Technology, vol. 20, no. 1, pp. 84-97, 2012.

[10] M. A. Stephens, C. Manzie, and M. C. Good, "Model predictive control for reference tracking on an industrial machine tool servo drive," IEEE Transactions on Industrial Informatics, vol. 9, no. 2, pp. 808-816, 2013.

[11] A. Zhakatayev, M. Rubagotti, and H. A. Varol, "Closed-loop control of variable stiffness actuated robots via nonlinear model predictive control," IEEE Access, vol. 3, pp. 235-248, 2015.

[12] Gurobi Optimization, Inc., Gurobi Optimizer Reference Manual, 2014. [Online]. Available: http://www.gurobi.com

[13] A. Bemporad and M. Morari, "Control of systems integrating logic, dynamics, and constraints," Automatica, vol. 35, no. 3, pp. 407-427, 1999.

[14] J. Širokỳ, F. Oldewurtel, J. Cigler, and S. Prívara, "Experimental analysis of model predictive control for an energy efficient building heating system," Applied Energy, vol. 88, no. 9, pp. 3079-3087, 2011.

[15] S. Prívara, J. Širokỳ, L. Ferkl, and J. Cigler, "Model predictive control of a building heating system: The first experience," Energy and Buildings, vol. 43, no. 2, pp. 564-572, 2011.

[16] Y. Ma, A. Kelman, A. Daly, and F. Borrelli, "Predictive control for energy efficient buildings with thermal storage," IEEE Control Systems Magazine, vol. 32, no. 1, pp. 44-64, 2012.

[17] F. Oldewurtel, A. Parisio, C. N. Jones, D. Gyalistras, M. Gwerder, V. Stauch, B. Lehmann, and M. Morari, "Use of model predictive control and weather forecasts for energy efficient building climate control," Energy and Buildings, vol. 45, pp. 15-27, 2012.

[18] T. Salsbury, P. Mhaskar, and S. J. Qin, "Predictive control methods to improve energy efficiency and reduce demand in buildings," Computers \& Chemical Engineering, vol. 51, pp. 77-85, 2013.

[19] S. Privara, J. Cigler, Z. Váňa, F. Oldewurtel, C. Sagerschnig, and E. Žáčeková, "Building modeling as a crucial part for building predictive control," Energy and Buildings, vol. 56, pp. 8-22, 2013.

[20] F. Oldewurtel, C. N. Jones, A. Parisio, and M. Morari, "Stochastic model predictive control for building climate control," IEEE Transactions on Control Systems Technology, vol. 22, no. 3, pp. 1198-1205, 2014.

[21] H. Farhangi, "A road map to integration: Perspectives on smart grid development," IEEE Power and Energy Magazine, vol. 12, no. 3, pp. 52-66, 2014.

[22] "Effekta Power Supplies Datasheet - BTL batteries," pp. 6061, 2014, http://pdf.effekta.com.de/Katalog_EN/Blaetterkatalog_EN/ Blaetterkatalog_EN.html.

[23] "Solar module series alfasolar Pyramid 60P - Datasheet," 2014, http: //en.alfasolar.biz/uploads/media/alfasolar-pyramid-60P-ENG-052014_ small_01.pdf.

[24] Z. Sun, Switched linear systems: control and design. Springer, 2006.

[25] L. Ljung, System identification: Theory for the user. Springer, 1998.

[26] F. Torrisi and A. Bemporad, "HYSDEL - A tool for generating computational hybrid models," IEEE Trans. Contr. Systems Technology, vol. 12, no. 2, pp. 235-249, Mar. 2004.

[27] G. Ferrari-Trecate, E. Gallestey, P. Letizia, M. Spedicato, M. Morari, and M. Antoine, "Modeling and control of co-generation power plants: a hybrid system approach," IEEE Transactions on Control Systems Technology, vol. 12, no. 5, pp. 694-705, 2004.

[28] D. Jingjing, S. Chunyue, and L. Ping, "Modeling and control of a continuous stirred tank reactor based on a mixed logical dynamical model," Chinese Journal of Chemical Engineering, vol. 15, no. 4, pp. 533-538, 2007.

[29] S. M. Sadat Kiaee, G. B. Gharehpetian, S. H. Hosseinian, and M. Abedi, "Home load and solar power management under real-time prices," in IEEE International Conference on Environment and Electrical Engineering, 2014, pp. 5-10.

[30] A. Bemporad, "Hybrid Toolbox - User's Guide," 2004, http://cse.lab. imtlucca.it/ bemporad/hybrid/toolbox. 